\title{
Research on the Influence of TikTok on Teenagers
}

\author{
Jiahong Guo
}

\author{
${ }^{1}$ Shanghai United International School-Wanyuan campus, Shanghai, 200001, China \\ *Corresponding author. Email: $18526203399 @ 163 . c o m$
}

\begin{abstract}
Nowadays, along with the vast development of social media, such as Twitter, Facebook, and YouTube. More and more new social networking softwares have come to the public's mind. For example, TikTok. TikTok is an application that is available for everyone to publish their videos, and the length of the video varies from 15 seconds to 1 minute. The videos include daily entertainment, talent show, popularization of knowledge and so on. Thanks to the reputation of TikTok, the videos on this platform spread quickly and bring its audience a sense of relief and a temporary escape from real life. However, along with the increase in the viewer group, more and more teenagers download this application and start to shoot videos at home, school, market and everywhere. People begin to argue that TikTok has brought too many negative effects to teenagers. In the research, there are both the positive and negative influences of TikTok. The object of this study is basically teenagers, and there will be different articles from the internet to compare and support the results. All the data are from CNKI, ScienceDirect, Taylor\& Francis Online, etc. The result is, TikTok played a nonnegligible impact on teenagers in three aspects---TikTok can be used as an educational tool, it can satisfy teenagers' mentality but it does contain over-exaggeration content that shapes teenager's value in a misleading way.
\end{abstract}

Keywords: Education, Teenager, TikTok, Social Media, Digital Cultures

\section{INTRODUCTION}

With the rise of digital media, people are investing more and more enthusiasm on the Internet. However, trends are always temporary. TikTok is undoubtedly one of the popular applications at present. Teenagers, as the majority of TikTok, have a new chance to express themselves on social media. In TikTok, millions of teenagers aged 12-20 years old share their short videos. There is a high chance that people will go viral just because of one video, and that is exactly what this kind of system does. It stimulates more and more people into the composition of short videos, hence leading to the new trend of shooting TikTok. Although TikTok is a private company, due to its optimistic development, people see the commercial opportunity of it and begin to explore potential human resources on TikTok. However, the negative aspects are still unclear to most people, since people only use their fragmented time to entertain themselves on the app, but the underlying effects of TikTok are one thing to consider as we dedicate our spare time to it[1]. So in the research paper, the negative effects of TikTok on teenagers will be analyzed. However, there are, of course, advantages to watching TikTok videos, so they are going to be included as well. As an extension, this paper will explore the relaxing and healing functions of TikTok on other people, apart from teenagers (this will be just a small portion of my research). Then, data will also be collected from classmates and friends (they are between the ages of 16-19), and the data will be analyzed using statistics to conclude the final result. Last but not the least, the purpose of this research is because people need to think more when they are watching short videos. If TikTok can bring people negative value, it will certainly offer valid value, and that is what people will hopefully focus on the next time they watch a video. Furthermore, because this is primarily for teenagers, and there is a mode called "teenager mode" that can send healthy and optimistic videos that motivate teenagers to cherish their current lives, there is a theory that discusses how this teenager mode may help to mitigate the negative impact TikTok has already had. Overall, the impact can be classified into educational, social, and perceptual aspects. With the understanding of the influence of TikTok, parents and teachers can better supervise teenagers so they can both enforce rules, such as when studying, TikTok and other social media are prohibited, but also create a positive 
relationship with teenagers. In addition, teenagers themselves can develop self-discipline by determining when to watch TikTok and what they should watch as they realize how their lives could be shaped by TikTok. Potentially, teenagers can use TikTok as a tool to enhance their self-esteem.

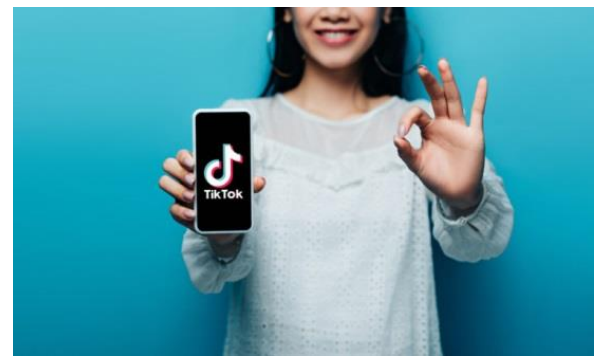

Figure 1: The balance of benefits and risks TikTok has brought to teens[2].

\section{ANALYSIS}

\subsection{Learning Tool}

To begin, many social media tools, such as TikTok, have provided an excellent opportunity for students to learn in an expression course. However, not only students benefit from online video platforms. In order to better understand how TikTok can influence teenagers, we must first lay eyes on how TikTok can shape the way teachers and professors giving their lectures to students. The research shows that scholars, academic administrator and university's professors also take advantage from TikTok, since they can search professional knowledge on TikTok, and there will always be someone that is an expert in that field, making a video to spread the knowledge.

Also, scholars can gain a reputation through TikTok. If people look closely when they click into a person's account, they will see a symbol that officially announces that the video maker is a professional scholar. There is usually a red circle with a check mark, and that will show the TikTok official has proved that the scholar is under authentication. In that case, when students surfing on the internet, or when they roll down to the next TikTok video, it will be a great chance that they can see these academic videos. In other words, this is the way how teenagers or school-aged students learn knowledge through online platforms, and most importantly in an easily acceptable and creative way. Also, students can like and share the videos their teachers make, so that the teacher will gain fame, and more students would be able to see the video. This would enhance a learning environment. Therefore, it is a double-win situation. However, TikTok's benefits are more than that.

On the other hand, TikTok stimulates students' talent in the creation of music. When the analysis focuses on the type of videos that are popular on TikTok, it conveys to the audience that these videos are composed of people' s movements with melodic music. This type of music video quickly becomes fashion, especially during the lockdown time due to COVID-19 outbreak. Since teenagers have nothing to do during the pandemic, they start to follow the trends on TikTok. People could look closely and take the advantage of it by exploiting the music into student's music courses, and hence different teaching and learning models(collaborative learning, mobile learning and so on) can be developed. In the music category, students can engage more in the learning process since if they shoot a video, they might need to practice several times behind the scene. In order to match their movement with music, they will be exposed to music, so the rhythm will be engraved into teenager's hearts and can even motivate and inspire their music composition. In addition, after teenagers filming the video, they will certainly need to edit the fragment of video. In such circumstances, they can even develop and enhance editing skills from TikTok. Due to the fact that there are various picture and video editing tools available on TikTok, waiting for teenager to study and use those, it facilitates the promotion of multimedia $\operatorname{art}[3]$.

Last but not the least, TikTok's music library enables people to edit an audio clip, mix it or add filters and stickers. In this instance, TikTok can be easily used as a learning tool since it gives people the great opportunity to get close to music and apply the music into practical usage.

\subsection{Self-Actualization \& Social Needs}

Furthermore, due to the fact that technology offers students an inspiring opportunity to use their time flexibly in terms of time, pace and place, TikTok can satisfy teenagers' mentality. The most popular trends on TikTok nowadays include dancing, scene play, covers of songs and people's fun observation in life. TikTok successfully attracts people in the whole world by sharing the same music under a trend, or simply inviting people's friends or family members to join TikTok and imitate the contents. Up to June 2020, Chinese internet short videos have a user scale that reaches 7.77 hundred million, and that is $94.5 \%$ of the whole internet user. In specific, teenager group comes in $14.8 \%$ of the entire online user. From this data, it is obvious that TikTok has taken the advantage of demographic dividends, and maximize its company's potential. Now, as for teenagers, since they are in puberty, they gradually perceive the world in both physical and mental ways. As they explore, their personality, perception and morality will begin to shape. Along with the sense of feeling that teenagers themselves are mature enough, when they are in 
puberty, they will have a period of time that they consider themselves as adults, so they also want other people to treat them as an individual human beings.

In this case, they would probably not follow the advice offered by their parents or elder family members. Instead, social media becomes a place where teenagers seek for a sense of identity and a sense of belonging. Therefore, teenagers realize their thinking system situation. In addition to that, puberty is the time that teenager's morality form, develop and become stable. When they play with TikTok, their multiple mental conditions can be fulfilled. First of all, TikTok satisfies the juvenile' s self-actualization. It is indeed TikTok's diverse vibe, handy functions make teenagers the chance to express themselves. As people observe some of the videos on TikTok, it will show that some teenagers like to reveal their positive speech and spread motivating theory with TikTok users, and hence gain people's attention and share their beliefs. Others, on the other hand, use TikTok as a way to promote their talents or skills, and that is way there are a good deal of dancing and singing videos on the online platform. However, there are some young adults that like to express their vulnerable mentality to their online friends, and they could also receive comfort and encouragement. As a result, teenagers can realize their self-actualization in different aspects and to a different degree. Moreover, since teenagers publish their videos for everyone to see, they will have more or fewer followers. And one of the most popular methods to use TikTok is to follow some of the followers back since people would gain stable attention.

Also, if TikTok detects that you are very active on TikTok, for example, if you post videos quite frequently, they will increase the probability of other people seeing you. In that case, a person's video can be seen by thousands or millions of people. There will always juveniles that share the same interests, and TikTok can bring these people contact each other. These teenagers who have common hobby and arrange an offline meeting, and they can gather around and form the traditional-called group. Also, since there are always present trends or buzzword spread by people in TikTok, when teenagers are at schools talking to their classmates, they can have new topics to discuss, so that it will reduce the alienation brought by different cultures. However, over $60 \%$ teenagers value TikTok simply as a tool to relax themselves, or reduce their academic or peer pressure. In school, due to various assignments such as oral presentations, group projects, or lab reports, students are put onto tons of pressure. Along with peer competition, if teenagers can have a temporary escape from studying in their spare time, TikTok can serve as a perfect app.

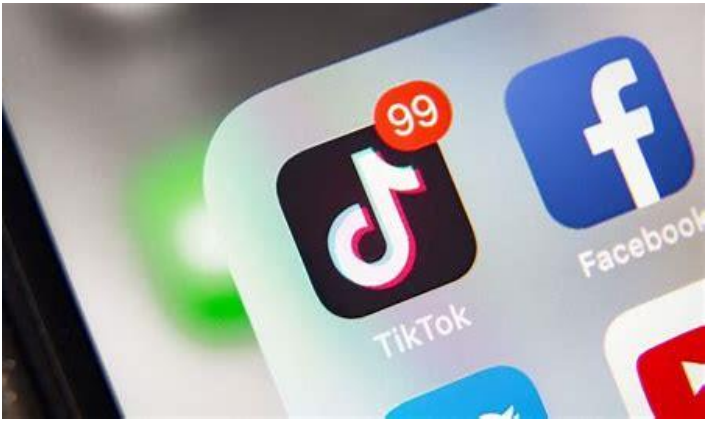

Figure 2: An everyday life illustration about why TikTok can achieve teenager's social demand[4].

\subsection{Aesthetic Fatigue}

Nevertheless, there are some side effects of using TikTok, such as blindly imitate video's content, aesthetic fatigue and biased value (how juveniles would view things)[4]. To start with, the most appealing function of TikTok is that it will detect what kind of video a person like, and the backstage supporter will send the same kind of video for that person to see. However, since the same videos will have exactly the same background music, and people will mimic the original video's content. This kind of video can easily gain hits in a short period of time, but the consequences are obvious. In addition, some of TikTok videos contain violent scenario or risky actions[5], if teenagers overuse TikTok, a minority of them would imitate the action and eventually lead to a tragedy. TikTok mislead teenagers and let them think it is easy to earn money, so that teenagers will focus less on the creativity and high-quality video making; instead, they would only follow the current trend and beg for some followers. Since social media make it easier to post video right now, everyone has the chance to become a vlogger. This circumstance stimulates the quantity of videos, but not the quality. In order to solve this problem, TikTok releases a function, in which if people like an original video, they can click in this bottom and they will have the same music and same filter and same everything with the primary video. This mechanism ensures that people are watching the videos they like. However, if TikTok users get exposed to the identical content of video over and over again, it is easier for teenagers to get tired of the genre of video they are watching[6].

Last but not the least, TikTok would warp teenagers , value of life. Admitted or not, the most popular video on TikTok is always debatable or argumentative. In this way, when people discuss about the video in comment section, it will also bring reputation to the video maker. However, the video might be vulgar or biased[7]. More commonly, there are people who show off their luxury items, or scatter his/ her money, and make a video about that[8]. This gives teenagers, who lack the independent thinking, a wrong perception that money is easy to gain and maintain. Most importantly, 
teenagers would think TikTok reveals the real world to them, but it turns out to be the opposite. TikTok users might have an extravagant life, but students must know the importance of study, instead of becoming internet influencers.

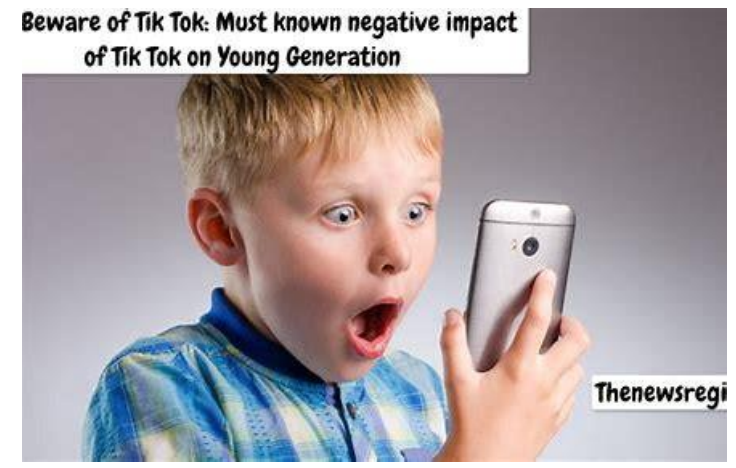

Figure 3: Potential detrimental ideology influenced by watching TikTok[9].

\section{CONCLUSION}

To summarize, as a part of the high sense of existence in teenagers' network world, TikTok has a profound influence on the youth, in an educational, social, and perceptual way. Although the majority of the effects it brought to the public were positive, some flaws should be noted. However, overall, TikTok provides people with a platform to express themselves. Without a doubt, it has changed people's ways of socializing, but TikTok still faces great challenges[10]. To correctly guide the public' $\mathrm{s}$ health development, TikTok should consciously assume public responsibility, and consider the advantages of short video transmission, and contribute to the publicity of culture[11]. To be specific, there are some improvements TikTok can make in the future. For instance, TikTok can force all minors to have name authentication, meaning teenagers under the age of 18 need to use their identification card to create an account on TikTok. In this way, both the TikTok management team and the teenagers' parents can better supervise the usage of TikTok and hence correct potential mistakes. On the other hand, TikTok already has a mode called juvenile mode, in which it will constantly filter unhealthy videos and make sure juveniles get to see learning and news videos. Also, TikTok could coerce teenagers to take a break when they've been endlessly using TikTok for more than two hours. After two hours, the app will automatically exit the interface, and teenagers will not be able to log in until tomorrow. In a word, since TikTok is one of the greatest social media software, it should take on the responsibility of promoting a positive guiding strategy, advocating the advantages of a short video, and finally contributing to the growth of teenagers' minds.

\section{REFERENCES}

[1]Greenhow, C. and Lewin, C., 2015. Social media and education: reconceptualizing the boundaries of formal and informal learning. Learning, Media and Technology, [online] 41(1), pp.6-30.

[2]Hinchliffe, T., 2021. Teens on TikTok: Do the Benefits Outweigh the Risks? [online] The Sociable.https://sociable.co/social-media/teens-ontiktok-do-the-benefits-outweigh-the-risks/[Accesse d 29 October 2021].

[3]Escamilla-Fajardo, P., Alguacil, M. and López-Carril, S., 2021. Incorporating TikTok in higher education: Pedagogical perspectives from a corporal expression sport sciences course. Journal of Hospitality, Leisure, Sport \& Tourism Education, 28, p.100302.

[4]Fish, G., 2021. why the first thing you should do with the TikTok app is uninstall it - [ weird things ].

[online]https://worldofweirdthings.com/2020/06/2

5/tiktok-app-security-spyware-china-privacy> [Accessed 29 October 2021].

[5]Meng, K. and Leung, L., 2021. Factors influencing TikTok engagement behaviors in China: An examination of gratifications sought, narcissism, and the Big Five personality traits. Telecommunications Policy, 45(7), p.102172.

[6]Scherr, S. and Wang, K., 2021. Explaining the success of social media with gratification niches: Motivations behind daytime, nighttime, and active use of TikTok in China. Computers in Human Behavior, 124, p.106893.

[7]Zeng Ying \&amp; Liu Yang. (2021). Negative effects of TikTok short video content and its guiding strategies. Media Forum (10), 40-41. doi:CNKI:SUN:CMLT.0.2021-10-019.

[8]Huang Wei Wei. (2021) TikTok's influence on young people and guidance strategy. Journal of Journalism Research (06), 79-80. doi:CNKI:SUN:XWDK.0.2021-06-038.

[9]The News Region. 2021. Beware of TikTok: Must know negative impact of TikTok on Young Generation - The News Region. [online] Available at:

https://thenewsregion.com/negative-impact-of-tik-t ok-on-young-generation/.

[10]Song Yang. (2021). The impact of short video on users' cognition from the perspective of visual communication. Audiovisual (09), 164-165. Doi: 10.19395/j.cnki.1674-246x. 2021.09.072.

[11]Tang Yue. Study on the impact of short video use on adolescent self-identity. 2021. Liaoning Normal University, MA thesis. 